International Journal of Modern Physics E

(C) World Scientific Publishing Company

\title{
Tree FCNC and non-unitarity of CKM matrix
}

\author{
C. S. Kim \\ Department of Physics, Yonsei University, Seoul, 120-749, Korea \\ cskim@yonsei.ac.kr \\ Amol Dighe \\ Tata Inst. of Fundamental Research, Mumbai 400005, India \\ amol@tifr.res.in \\ Received (received date) \\ Revised (revised date)
}

\begin{abstract}
We discuss possible signatures of the tree level FCNC, which results from the nonunitarity of CKM matrix. We first define the unitaity step-by-step, and possible test of the non-unitaity through the 4-value-KM parametrization. We, then, show how the phase angle of the unitary triangle would change in case of the vector-like down quark model. As another example of tree FCNC, we investigate the leptophobic $Z^{\prime}$ model and its application to the recent $B_{s}$ mixing measurements.
\end{abstract}

\section{Introduction}

The flavor sector of the standard model (SM) is not yet properly understood, and in particular, the mechanism of charge-parity $(C P)$ violation still eludes us. The Cabbibo-Kobayashi-Maskawa $(\mathrm{CKM})$ matrix $^{1}$ parametrizes the mixing between the three quark famlies, and provides the the only source of $C P$ violation within the model. This is a very strong prediction of the SM. While it is consistent with the observations till now, it is important to look for any sources of $C P$ violation beyond the SM and the observable signals they may manifest themselves in.

Here, in particular, we investigate the possibility of a non-unitary CKM matrix. The unitarity of the CKM matrix in SM by virtue of the fact that there are three quarks each of up and down type. Though it is the most parsimonious explanation still consistent with all the available data, the presence of more quarks that interact significantly with the existing ones has not been ruled out. Most of the analyses of the quark matrix have been carried out in the context of a $3 \times 3$ unitary CKM matrix, the fits having been performed to the parameters that implicitly assume the $3 \times 3$ unitarity. We currently have only a few measurements available to test this assumption directly. However, future measurements from the B factories and the hadronic machines will provide us with means of testing this assumption and/or constraining the non-unitary contributions. 
The $3 \times 3$ CKM matrix can be non-unitary if it is an effective mixing matrix, i.e. if it is a submatrix of a larger matrix. This larger matrix may be, for instance, a $n \times n$ unitary matrix representing the mixing between $n$ generations, or a $n \times m$ mixing matrix that is only partially unitary ${ }^{\mathrm{a}}$ like the one that arises in models with extra isosinglet bottom quarks. Here we consider in detail the model with a single "Vector-like down quark" $(\mathrm{VdQ})^{2}$, in which only one isosinglet down quark is added to the SM. Here the CKM matrix gets modified to a $3 \times 4$ matrix $V_{V d Q}$. Vector-like fermions appear in many extensions of the SM, like the low energy limit of $E_{6}$ GUT models ${ }^{3}$, or models with extra spatial dimensions on the TeV scale ${ }^{4}$ as towers vector-like fermions of Kaluza-Klein excitations for the SM quarks. The detailed phenomenological studies on the possible FCNC effects, the violation of GIM mechanism, $\Delta \rho$ constraints and $B \rightarrow X_{s} \gamma$ decays from those towers of vectorlike quaks have been investigated in $\operatorname{Ref}^{5}$.

\section{A Flexible Parametrization of CKM matrix via 4VKM}

The Cabibbo-Kobayashi-Maskawa $(\mathrm{CKM})^{1}$ matrix makes us possible to explain all flavor changing weak decay processes and CP violating phenomena up to now. Unitarity of the CKM matrix in the standard model (SM) is a unique property that we cannot loosen. We can use any parametrization of the CKM matrix as long as its unitarity is conserved. The original parametrization for three generation quark mixing is the Kobayash-Maskawa (KM) parametrization. The standard parametrization proposed by Chau and Keung ${ }^{6}$ is the product of three complex rotation matrices which are characterized by the three Euler angles, $\theta_{12}, \theta_{13}, \theta_{23}$ and an $\mathrm{CP}$-violating phase $\delta_{13}$. More widely used one is the Wolfenstein parametrization ${ }^{7}$, which was suggested as a simple expansion of the CKM matrix in terms of the four parameters: $\lambda, A, \rho$ and $\eta$. It has been also known that the CKM matrix for the three-generation case can be parameterized in terms of the moduli of four of its elements ${ }^{8}$. This fourvalue-KM (4VKM) parametrization is rephasing invariant and directly related to the measured quantities. In three generation case we always need four independent parameters to define a unitary $3 \times 3$ matrix, as explained, eg. $\theta_{12}, \theta_{13}, \theta_{23}$ and $\delta_{13}$, or $\lambda, A, \rho$ and $\eta$ or even only moduli of any four independent elements of the matrix.

The 4VKM parametrization has several advantages over the other parametrization. This parametrization doesn't need any specific representations for the mixing angles as long as the CKM is unitary, and no ambiguity over the definition of its complex CP phase is present above all. Secondly, the Jarlskog invariant quantity $J_{c p}$ and non-trivial higher invariants can be reformulated as functions of moduli and quadri-products ${ }^{9}$. However, in the $4 \mathrm{VKM}$ parametrization initial four-moduli input values should be fixed by experiments. Once we set four moduli to specific

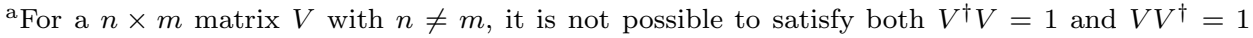
simultaneously. 
values, remaining five moduli of mixing elements are automatically fixed and we may lose some characteristic effects from interplaying between the moduli.

Many groups have made global fits and numerical works on CKM matrix elements with conventional representations which satisfy unitarity ${ }^{10}$. One of the problems in these conventional parameterizations is that they are fully and completely unitary and are not flexible to include possible non-unitary properties resulted from unknown new physics. Therefore, it is a complicate task to make a step-by-step test to check the unitarity with experimental data if you use a unitary parametrization. In the following, we present three extended definitions for the unitarities of mixing matrix $V$ in the order of the strength of the constraints:

- Weak Unitary Conditions (WUC): We define that the mixing matrix $V$ is weak unitary if it satisfies

$$
\sum_{\alpha}\left|V_{i \alpha}\right|^{2}=\sum_{j}\left|V_{j \beta}\right|^{2}=1 \text { for all } i=u, c, t, \text { and } \quad \beta=d, s, b .
$$

These constraints appear to be well satisfied experimentally for the three generation case, and we start from this. Actually it was pointed out that there is an apparent functional violation in the available data: $\left|V_{u d}\right|^{2}+$ $\left|V_{u s}\right|^{2}+\left|V_{u b}\right|^{2}<1^{11}$. For such a case with $\sum_{\alpha}\left|V_{u \alpha}\right|^{2}=a<1$, we can easily generalize our method, and we just start with this new condition.

- Almost Unitary Conditions (AUC): In addition to the constraint Eq. (1), if the following constraints are satisfied

$$
\begin{array}{r}
\sum_{\alpha, i \neq j} V_{i \alpha}^{*} V_{j \alpha}=\sum_{j, \alpha \neq \beta} V_{j \alpha}^{*} V_{j \beta}=0 \text { for some parts of } \quad i, j=u, c, t, \\
\text { and } \quad \alpha, \beta=d, s, b,
\end{array}
$$

let us call the mixing matrix almost unitary. Some combinations, which do not satisfy Eq. (2), may not make closed triangles, and may have different areas even though making closed triangles. We have no specific models in which the mixing matrix satisfies this almost unitary conditions. Therefore, we will not consider the case with AUC.

- Full Unitary Conditions (FUC): This corresponds to usual unitarity in which Eqs. (1), (2) are satisfied for all the indices. All six unitarity triangles from Eq. (2) have the same areas.

As a next step, we further assume that the mixing matrix $V$ satisfies full unitary conditions. Then we have six more constraints:

$$
\begin{aligned}
\sum_{j=d, s, b} V_{i j} V_{k j}^{*} & =0, & (i, k) & =(u, c),(u, t),(c, t), \\
\sum_{j=u, c, t} V_{j i} V_{j k}^{*} & =0, & (i, k) & =(d, s),(d, b),(s, b) .
\end{aligned}
$$

These constraints cannot be represented without introduction of complex numbers analytically. If we know all the absolute values of $V$, however, we can express 
necessary and sufficient conditions for the constraints, Eqs. (3), in a geometric way. Eqs. (3) give six unitarity triangles corresponding to each six constraints, and all six triangles have equal area that is directly related to the Jarlskog's rephasing invariant parameter $J_{C P}$. If we take one of the constraints Eqs. (3), for example,

$$
\sum_{j=u, c, t} V_{j d} V_{j b}^{*}=0
$$

a triangle is composed of three sides with lengths $\left|V_{u d}\right|\left|V_{u b}\right|,\left|V_{c d}\right|\left|V_{c b}\right|$, and $\left|V_{t d}\right|\left|V_{t b}\right|$, with a necessary condition

$$
\left|V_{c d}\right|\left|V_{c b}\right| \leq\left|V_{u d}\right|\left|V_{u b}\right|+\left|V_{t d}\right|\left|V_{t b}\right|
$$

where the equality holds in $\mathrm{CP}$ conserving case. For more general argument, let us rewrite Eq. (5) as follows:

$$
l_{2} \leq l_{1}+l_{3},
$$

where, as an example, $l_{1}=\left|V_{u d}\right|\left|V_{u b}\right|, l_{2}=\left|V_{c d}\right|\left|V_{c b}\right|$, and $l_{3}=\left|V_{t d}\right|\left|V_{t b}\right|$. After taking the square on both sides of Eq. (6) we can rearrange the constraint equation as follows:

$$
f\left(l_{1}, l_{2}, l_{3}\right) \equiv 2 l_{1}^{2} l_{2}^{2}+2 l_{2}^{2} l_{3}^{2}+2 l_{1}^{2} l_{3}^{2}-l_{1}^{4}-l_{2}^{4}-l_{3}^{4} \geq 0,
$$

where we denote newly introduced function $f$ for later use. Using the Heron's formula, the square of triangular area can be rewritten as follows:

$$
A^{2}=s\left(s-l_{1}\right)\left(s-l_{2}\right)\left(s-l_{3}\right)=\frac{1}{16} f\left(l_{1}, l_{2}, l_{3}\right),
$$

where $s=\left(l_{1}+l_{2}+l_{3}\right) / 2$. So the necessary condition (7) for the complete triangle means non-negative value of $A^{2}$. The Jarlskog's invariant parameter is written as follows:

$$
J_{C P}=2 A=\frac{1}{2} \sqrt{f\left(l_{1}, l_{2}, l_{3}\right)} .
$$

Three angles $\alpha, \beta, \gamma$ of the unitarity triangle, which characterize $\mathrm{CP}$ violation, are defined as follows:

$$
\begin{aligned}
& \alpha=\operatorname{Arg}\left[-\left(V_{t d} V_{t b}^{*}\right) /\left(V_{u d} V_{u b}^{*}\right)\right], \\
& \beta=\operatorname{Arg}\left[-\left(V_{c d} V_{c b}^{*}\right) /\left(V_{t d} V_{t b}^{*}\right)\right], \\
& \gamma=\operatorname{Arg}\left[-\left(V_{u d} V_{u b}^{*}\right) /\left(V_{c d} V_{c b}^{*}\right)\right] .
\end{aligned}
$$

The sum of those three angles, defined as the intersections of three lines, would be always equal to $180^{\circ}$, even though the three lines may not be closed to make a triangle, i.e. in case that CKM matrix is not unitary at all. We can also define these quantities from the area of the unitary triangle and its sides:

$$
\begin{aligned}
\sin \beta^{\prime} & =\frac{2 A}{\left|V_{t d}\right|\left|V_{t b}\right|\left|V_{c d}\right|\left|V_{c b}\right|}, \\
\sin \gamma^{\prime} & =\frac{2 A}{\left|V_{u d}\right|\left|V_{u b}\right|\left|V_{c d}\right|\left|V_{c b}\right|}, \\
\alpha^{\prime} & =\pi-\beta^{\prime}-\gamma^{\prime},
\end{aligned}
$$


when the FUC is fully satisfied and the area of the triangles can be defined from (8). Any experimental data that indicates $\alpha \neq \alpha^{\prime}$ or $\beta \neq \beta^{\prime}$ or $\gamma \neq \gamma^{\prime}$ means that three generation quark mixing matrix $V$ is not fully unitary.

\section{3. $B$ Meson Mixing Phase in Vector-like Down Quark Model}

The CKM matrix $\left(V_{C K M}\right)$ is unitary, i.e. it satisfies

$$
\left(V_{C K M}\right)^{\dagger} V_{C K M}=V_{C K M}\left(V_{C K M}\right)^{\dagger}=1 .
$$

The unitarity relations satisfied by the CKM matrix elements lead to three independent unitarity triangles with equal areas. (The equality of the areas reflects that there is only one source of $C P$ violation in the SM). The measurements of the angles of these triangles provide powerful tests for the unitarity of the CKM matrix. After using the constraints in (16) and exploiting the freedom to change the relative phases of individual quarks, $V_{C K M}$ can be parametrized with 3 real parameters and 1 complex phase parameter.

The matrix $V_{V d Q}$ satisfies

$$
V_{V d Q}\left(V_{V d Q}\right)^{\dagger}=1
$$

After exploiting the freedom to change the relative phases of individual quarks, $V_{V D Q}$ can be completely described in terms of 6 real parameters and 3 complex phase parameters. The extra complex phases indicate the possibility of extra sources of $C P$ violation. The limits on some of these extra parameters have already been computed in $\operatorname{Ref}^{2}$.

Here we concentrate on the constraints that come from the mixing phases measured in the $B_{d}-\bar{B}_{d}$ and $B_{s}-\bar{B}_{s}$ systems. In the SM, these phases correspond respectively to the angles

$$
\beta \equiv \operatorname{Arg}\left(-\frac{\mathrm{V}_{\mathrm{cb}}^{*} \mathrm{~V}_{\mathrm{cd}}}{\mathrm{V}_{\mathrm{tb}}^{*} \mathrm{~V}_{\mathrm{td}}}\right), \chi \equiv \operatorname{Arg}\left(-\frac{\mathrm{V}_{\mathrm{cb}}^{*} \mathrm{~V}_{\mathrm{cs}}}{\mathrm{V}_{\mathrm{tb}}^{*} \mathrm{~V}_{\mathrm{ts}}}\right)
$$

of the unitarity triangles. In particular, the time-dependent $C P$ asymmetry in $B_{d}(t) \rightarrow J / \psi K_{S / L}$ measures $\sin (2 \beta)$ and the the time-dependent $C P$ asymmetry in $B_{s}(t) \rightarrow J / \psi \eta^{\left({ }^{\prime}\right)}$ or $B_{s}(t) \rightarrow J / \psi \phi$ would give $\sin (2 \chi)$ (after taking care of the significant lifetime difference in the $B_{s}$ system). Whereas $\sin (2 \beta)$ has already been measured at the $\mathrm{B}$-factories ${ }^{12}$, the value of $\sin (2 \chi)$, predicted to be small in the SM $(\chi \approx-0.015)$, has not yet been measured.

One of the clearest signals of new physics sources of $C P$ violation would be a value of $\chi$ that is much higher than the SM prediction. In this paper, we shall examine if such a large value of $\chi$ is possible under the VdQ model.

\subsection{The parametrization}

The 6 real and 3 phase parameters that describe the quark mixing matrix in the VdQ model can be chosen to be the six rotation angles $\theta_{i j},(1 \leq i<j \leq 4)$ and 
three phases $e^{i \delta_{X}}, X \in\left\{u b, u b^{\prime}, c b^{\prime}\right\}$. Here we denote the isosinglet down quark by $b^{\prime}$. The matrix $V_{V d Q}$ can be written as

$$
V_{V d Q}=K \cdot V_{4 G},
$$

where

$$
K=\left(\begin{array}{llll}
1 & 0 & 0 & 0 \\
0 & 1 & 0 & 0 \\
0 & 0 & 1 & 0
\end{array}\right)
$$

and $V_{4 G}$ is the extension of $V_{C K M}$ for four generations:

$$
\begin{aligned}
V_{4 G} \equiv & R_{34}\left(\theta_{34}\right) \cdot \Phi\left(0,-\delta_{c b^{\prime}}, 0,0\right) \cdot R_{24}\left(\theta_{24}\right) \cdot \Phi\left(-\delta_{u b^{\prime}}, \delta_{c b^{\prime}}, 0,0\right) \cdot \\
& \cdot R_{14}\left(\theta_{14}\right) \cdot \Phi\left(\delta_{u b^{\prime}}, 0,0,0\right) \cdot R_{23}\left(\theta_{23}\right) \cdot \Phi\left(-\delta_{u b}, 0,0,0\right) \\
& \cdot R_{13}\left(\theta_{13}\right) \cdot \Phi\left(0,0, \delta_{u b}, 0\right) \cdot R_{12}\left(\theta_{12}\right)
\end{aligned}
$$

Here $R_{i j}$ represents the rotation in $i-j$ plane, and $\Phi\left(\delta_{1}, \delta_{2}, \delta_{3}, \delta_{4}\right)$ is the diagonal matrix with diagonal elements $e^{i \delta_{i}}, 1 \leq i \leq 4$. Note that $V_{V d Q}$ is just $V_{4 G}$ with its fourth row removed. Also, putting $\theta_{14}=\theta_{24}=\theta_{34}=0$ reduce $V_{4 G}$ as well as $V_{V d Q}$ to a $3 \times 3$ submatrix $V_{C K M}$ and zeroes as the remaining elements.

Taking a clue from the hierarchy of the measured mixing angles, let us use the sine of the Cabbibo angle, $\lambda \approx 0.22$, as the expansion parameter. The angles already present in the CKM matrix are

$$
\sin \left(\theta_{12}\right) \equiv \lambda, \quad \sin \left(\theta_{23}\right) \equiv A \lambda^{2}, \quad \sin \left(\theta_{13}\right) \equiv A C \lambda^{3} .
$$

Keeping with the philosophy of expanding in $\lambda$, we shall parametrize the new physics angles as

$$
\sin \left(\theta_{14}\right) \equiv p \lambda^{3}, \quad \sin \left(\theta_{24}\right) \equiv q \lambda^{2}, \quad \sin \left(\theta_{34}\right) \equiv r \lambda .
$$

This reflects the assumption that the mixing between two generations follows a hierarchical pattern, i.e. the further apart the generations, the smaller the mixing between them.

We expand the elements of $V_{V d Q}$ in powers of $\lambda$ such that each element is accurate upto a multiplicative factor of $\left.\left[1+\mathcal{O}\left(\lambda^{3}\right)\right]\right)$. The definitions of the parameters in (22) and (23) translate to

$$
\begin{array}{lll}
V_{u s}=\lambda, & V_{c b}=A \lambda^{2}, & V_{u b}=A \lambda^{3} C e^{-i \delta_{u b}}, \\
V_{u b^{\prime}}=p \lambda^{3} e^{-i \delta_{u b^{\prime}}}, & V_{c b^{\prime}}=q \lambda^{2} e^{-i \delta_{c b^{\prime}}}, & V_{t b^{\prime}}=r \lambda .
\end{array}
$$


These give rise to the other elements of $V_{V d Q}$ as:

$$
\begin{aligned}
V_{u d}= & 1-\frac{\lambda^{2}}{2}+\mathcal{O}\left(\lambda^{4}\right) \\
V_{c d}= & -\lambda+\mathcal{O}\left(\lambda^{5}\right) \\
V_{c s}= & 1-\frac{\lambda^{2}}{2}+\mathcal{O}\left(\lambda^{4}\right) \\
V_{t d}= & A \lambda^{3}\left(1-C e^{i \delta_{u b}}\right)+r \lambda^{4}\left(q e^{i \delta_{c b^{\prime}}}-p e^{i \delta_{u b^{\prime}}}\right) \\
& +\frac{A}{2} \lambda^{5}\left(-r^{2}+\left(C+C r^{2}\right) e^{i \delta_{u b}}\right)+\mathcal{O}\left(\lambda^{6}\right) \\
V_{t s}= & -A \lambda^{2}-q r \lambda^{3} e^{i \delta_{c b^{\prime}}}+\frac{A}{2} \lambda^{4}\left(1+r^{2}-2 C e^{i \delta_{u b}}\right)+\mathcal{O}\left(\lambda^{5}\right) \\
V_{t b}= & 1-\frac{r^{2} \lambda^{2}}{2}+\mathcal{O}\left(\lambda^{4}\right)
\end{aligned}
$$

Note that in the limit $p=q=r=0$, only the elements present in $V_{C K M}$ retain nonvanishing values, and the above expansion corresponds to the Wolfenstein parametrization ${ }^{7}$ with $C=\sqrt{\rho^{2}+\eta^{2}}$ and $\delta_{u b}=\tan ^{-1}(\eta / \rho)$.

The matrix $V_{V d Q}$ is also parametrized often (e.g. see Ref ${ }^{2}$ ) in terms of the parameters (in addition to the usual CKM parameters)

$$
\begin{array}{lll}
D_{d}^{2}=\left|V_{t^{\prime} d}\right|^{2}, & D_{s}^{2}=\left|V_{t^{\prime} s}\right|^{2}, & D_{b}^{2}=\left|V_{t^{\prime} b}\right|^{2}, \\
U_{s d}=-V_{t^{\prime} s}^{*} V_{t^{\prime} d} & U_{b s}=-V_{t^{\prime} b}^{*} V_{t^{\prime} s} & U_{b d}=-V_{t^{\prime} b}^{*} V_{t^{\prime} d}
\end{array}
$$

where the elements $V_{t^{\prime} q}$ are given in our notation by

$$
\begin{aligned}
V_{t^{\prime} d}= & \lambda^{3}\left(q e^{i \delta_{c b^{\prime}}}-p e^{i \delta_{u b^{\prime}}}\right)+A r \lambda^{4}\left(1+C e^{i \delta_{u b}}\right) \\
& +\frac{\lambda^{5}}{2}\left(p e^{i \delta_{u b^{\prime}}}-q r^{2} e^{i \delta_{c b^{\prime}}}+p r^{2} e^{i \delta_{u b^{\prime}}}\right)+\mathcal{O}\left(\lambda^{6}\right) \\
V_{t^{\prime} s}= & q \lambda^{2} e^{i \delta_{c b^{\prime}}}+A r \lambda^{3} \\
& +\lambda^{4}\left(-p e^{i \delta_{u b^{\prime}}}+\frac{q}{2} e^{i \delta_{c b^{\prime}}}+\frac{q r^{2}}{2} e^{i \delta_{c b^{\prime}}}\right)+\mathcal{O}\left(\lambda^{5}\right) \\
V_{t^{\prime} b}= & -r \lambda+\mathcal{O}\left(\lambda^{4}\right) \\
V_{t^{\prime} b^{\prime}}= & 1-\frac{r^{2} \lambda^{2}}{2}+\mathcal{O}\left(\lambda^{4}\right)
\end{aligned}
$$

We already have strong direct bounds on the magnitudes of the elements of the CKM matrix. From Ref ${ }^{13}$, we can derive

$$
0.216<\lambda<0.223,0.76<A<0.90,0.23<C<0.59 .
$$

at $90 \%$ C.L. from the direct measurements of $\left|V_{u s}\right|,\left|V_{c b}\right|$ and $\left|V_{u b} / V_{c b}\right|$, which do not assume the unitarity of $V_{C K M}$. Combining the direct measurements of the magnitudes of the elements in the first two rows with the unitarity constraints (17), we get the $90 \%$ C.L. bounds on $\left|V_{u b^{\prime}}\right|$ and $\left|V_{c b^{\prime}}\right|$ as

$$
\left|V_{u b^{\prime}}\right|<0.094, \quad\left|V_{c b^{\prime}}\right|<0.147 .
$$


These correspond to $p<9.0, q<3.05$.

In addition, a strong constraint is obtained on the combination $X_{b b}^{L} \equiv$ $\left(V_{C K M}^{\dagger} V_{C K M}\right)_{b b}$ through the measurements involving $Z \rightarrow b \bar{b}$ : we have $X_{b b}^{L}=$ $0.996 \pm 0.005^{14}$. This translates to $\left|V_{t b^{\prime}}\right|<0.11$ at $90 \%$ C.L., which corresponds to $r<0.5$.

\subsection{Mixing phases in the VdQ model}

In the VdQ model, the box diagrams that contributes the phase to the $B_{d}-\bar{B}_{d}$ and $B_{s}-\bar{B}_{s}$ mixing are the same as those in the SM. Therefore, the mixing phases are simply

$$
\tilde{\beta} \equiv \operatorname{Arg}\left(-\frac{\tilde{\mathrm{V}}_{\mathrm{cb}}^{*} \tilde{\mathrm{V}}_{\mathrm{cd}}}{\tilde{\mathrm{V}}_{\mathrm{tb}}^{*} \tilde{\mathrm{V}}_{\mathrm{td}}}\right), \quad \tilde{\chi} \equiv \operatorname{Arg}\left(-\frac{\tilde{\mathrm{V}}_{\mathrm{cb}}^{*} \tilde{\mathrm{V}}_{\mathrm{cs}}}{\tilde{\mathrm{V}}_{\mathrm{tb}}^{*} \tilde{\mathrm{V}}_{\mathrm{ts}}}\right) .
$$

We use the superscript ${ }^{\sim}$ to denote the quantities in the VdQ model.

\subsubsection{Unitarity relations involving $\beta$ and $\tilde{\beta}$}

In the standard model, the unitarity (16) of the $3 \times 3$ CKM matrix implies unitarity triangle relations. The unitarity constraints $(17)$ on the $3 \times 4$ matrix $V_{V d Q}$ do not lead to any unitarity triangles. However, the $3 \times 3$ unitarity triangles get modified in a predictable and controlled manner. We demonstrate this in this subsection and the next.

Let us first start with the "standard" unitarity triangle in the SM, which arises from the equation

$$
V_{u b}^{*} V_{u d}+V_{c b}^{*} V_{c d}+V_{t b}^{*} V_{t d}=0
$$

which is true in the SM. The angles of this unitarity triangle (Fig. 1) are defined as

$$
\alpha \equiv \operatorname{Arg}\left(-\frac{\mathrm{V}_{\mathrm{tb}}^{*} \mathrm{~V}_{\mathrm{td}}}{\mathrm{V}_{\mathrm{ub}}^{*} \mathrm{~V}_{\mathrm{ud}}}\right), \beta \equiv \operatorname{Arg}\left(-\frac{\mathrm{V}_{\mathrm{cb}}^{*} \mathrm{~V}_{\mathrm{cd}}}{\mathrm{V}_{\mathrm{tb}}^{*} \mathrm{~V}_{\mathrm{td}}}\right), \gamma \equiv \operatorname{Arg}\left(-\frac{\mathrm{V}_{\mathrm{ub}}^{*} \mathrm{~V}_{\mathrm{ud}}}{\mathrm{V}_{\mathrm{cb}}^{*} \mathrm{~V}_{\mathrm{cd}}}\right) .
$$

A related unitarity relation in the $\mathrm{VdQ}$ model is

$$
\tilde{V}_{u d}^{*} \tilde{V}_{t d}+\tilde{V}_{u s}^{*} \tilde{V}_{t s}+\tilde{V}_{u b}^{*} \tilde{V}_{t b}+\tilde{V}_{u b^{\prime}}^{*} \tilde{V}_{t b^{\prime}}=0
$$

which may be called a "quadrilateral" relation. This quadrilateral may be superimposed on the SM unitarity triangle as shown in Fig. 1.

The following things should be noted. The bases of the triangle and the quadrilateral make an angle $\operatorname{Arg}\left[\left(\tilde{V}_{c b}^{*} \tilde{V}_{c d}\right) /\left(\tilde{V}_{u s}^{*} \tilde{V}_{t s}\right)\right]$ with each other, which is equal to $\tilde{\chi}$. The "left" edges of the two figures coincide since $\operatorname{Arg}\left(\tilde{V}_{u b}^{*} \tilde{V}_{t b}\right)=\operatorname{Arg}\left(V_{u b}^{*} V_{u d}\right)=\delta_{u b}$.

The measurements of the $C P$ asymmetry in the decay $B_{d}(t) \rightarrow J / \psi K_{S / L}$ at the $B$ factories ${ }^{12}$ measure $\sin \left(2 \beta_{\psi K}\right)=\sin (2 \tilde{\beta})=0.734 \pm 0.054$. In Fig. $1, \tilde{\beta}$ is the angle made by the "right" leg of the quadrilateral with the real axis. 


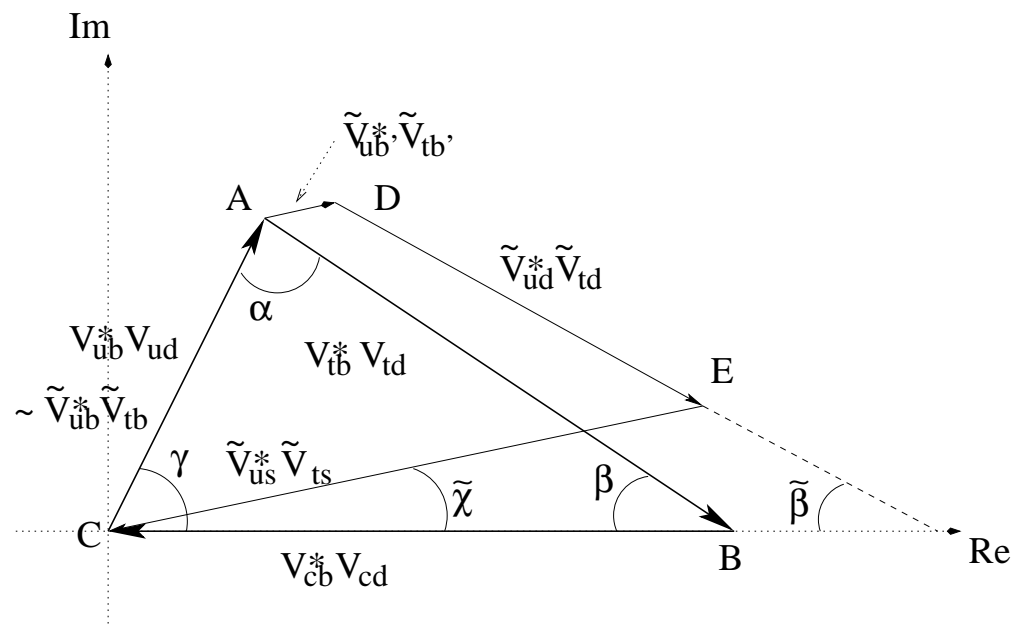

Fig. 1. The unitarity triangle (ABC) in the $\mathrm{SM}$ with angles $\alpha, \beta, \gamma$ and the corresponding unitarity quadrilateral (ACED) in the VdQ model.

We can estimate the systematic error in the measurement of $\beta$ due to the VdQ extension to be

$$
\begin{aligned}
\Delta \beta & \equiv \tilde{\beta}-\beta=\operatorname{Arg}\left(\frac{\tilde{V}_{u d}^{*} \tilde{V}_{t d}}{V_{t b}^{*} V_{t d}}\right)=\operatorname{Arg}\left(\frac{\tilde{V}_{t d}}{V_{t d}}\right) \\
& \approx \operatorname{Arg}\left[1+\frac{r \lambda}{A}\left(\frac{q e^{i \delta_{c b^{\prime}}}-p e^{i \delta_{u b^{\prime}}}}{1-C e^{i \delta_{u b}}}\right)\right] .
\end{aligned}
$$

Thus, we estimate $\Delta \beta \lesssim \lambda$. Since the current experimental error corresponds to $\Delta \beta \approx 0.04$, the deviation due to the $\mathrm{VdQ}$ extension may be important. It is, however, not possible to identify this deviation from the measurement of $\beta_{\psi K}$ alone.

\subsubsection{Unitarity relations involving $\chi$ and $\tilde{\chi}$}

The "squashed" unitarity triangle in the SM arises from the equation

$$
V_{u b}^{*} V_{u s}+V_{c b}^{*} V_{c s}+V_{t b}^{*} V_{t s}=0
$$

The angles of this unitarity triangle (Fig. 2) are

$$
\chi \equiv \operatorname{Arg}\left(-\frac{\mathrm{V}_{\mathrm{cb}}^{*} \mathrm{~V}_{\mathrm{cs}}}{\mathrm{V}_{\mathrm{tb}}^{*} \mathrm{~V}_{\mathrm{ts}}}\right), \Theta \equiv \operatorname{Arg}\left(-\frac{\mathrm{V}_{\mathrm{tb}}^{*} \mathrm{~V}_{\mathrm{ts}}}{\mathrm{V}_{\mathrm{ub}}^{*} \mathrm{~V}_{\mathrm{us}}}\right)=\gamma-\chi, \pi-\Theta-\chi .
$$

Unitarity of the CKM matrix forces the angle $\chi$ (also called in the literature by various names like $\delta \phi, \phi_{s}, 2 \delta \gamma, \beta_{s}$ ) to be very small: in fact, the relation

$$
\sin \chi \approx\left|\frac{V_{u s}}{V_{u d}}\right|^{2} \frac{\sin \beta \sin (\gamma+\chi)}{\sin (\beta+\gamma)}\left[1+\mathcal{O}\left(\lambda^{4}\right)\right]
$$






Fig. 2. The "squashed" unitarity triangle (PQR) in the SM and the corresponding unitarity quadrilateral (QRST) in the VdQ model.

is a true test of unitarity (as opposed to the relation $\alpha+\beta+\gamma=\pi$, which is trivially true by the definitions in (40)). This gives $\chi \approx 0.015$ in the SM. If the value of $\chi$ (which also is the $B_{s}-\bar{B}_{s}$ mixing phase) is indeed this small, it may be hard to measure it even at the LHC. However, as we shall see, in the extensions of the SM that defy $3 \times 3$ CKM unitarity, it is indeed possible to get higher values of this phase.

The unitarity "quadrilateral" relation in the VdQ models that is relevant here is

$$
\tilde{V}_{c d}^{*} \tilde{V}_{t d}+\tilde{V}_{c s}^{*} \tilde{V}_{t s}+\tilde{V}_{c b}^{*} \tilde{V}_{t b}+\tilde{V}_{c b^{\prime}}^{*} \tilde{V}_{t b^{\prime}}=0,
$$

This quadrilateral may be superimposed on the SM unitarity triangle as shown in Fig. 2. The bases of both the triangle and the quadrilateral coincide since $\operatorname{Arg}\left(\tilde{V}_{c b}^{*} \tilde{V}_{t b}\right)=\operatorname{Arg}\left(V_{c b}^{*} V_{c s}\right)=0$.

The systematic error introduced in the measurement of $\chi$ due to the VdQ extension is

$$
\begin{aligned}
\Delta \chi & \equiv \tilde{\chi}-\chi=\operatorname{Arg}\left(\frac{\tilde{V}_{c s}^{*} \tilde{V}_{t s}}{V_{t b}^{*} V_{t s}}\right)=\operatorname{Arg}\left(\frac{\tilde{V}_{t s}}{V_{t s}}\right) \\
& \approx \operatorname{Arg}\left(1+\frac{q r \lambda}{A} e^{i \delta_{c b^{\prime}}}\right) \sim \frac{q r \lambda}{A} .
\end{aligned}
$$

This, $\Delta \chi$ (or $\tilde{\chi})$ can be as large as the order of $\lambda \approx 0.2$. In this case, the contribution due to the VdQ extension dominates over the SM contribution by about an order of magnitude. If the value of $\tilde{\chi}$ is indeed this high, it can be definitely measured at the LHC or perhaps even at the Tevatron.

At the same time, the value of $\tilde{\chi}$ is restricted to be $\mathcal{O}(\lambda)$. Thus, too large a measured value of the $B_{s}-\bar{B}_{s}$ mixing phase will be able to rule out the VdQ model.

The two major modes employed by the $B$ factories for the measurement of the $B_{d}-\bar{B}_{d}$ mixing phase are $B_{d}(t) \rightarrow J / \psi K_{S / L}$ and $B_{d}(t) \rightarrow \phi K_{S / L}$. The central values 
of the phases obtained through these two modes currently differ a lot from each other, however these values are still consistent, given large statistical errors on the measurement of the phase through $\phi K_{S / L}$.

The time dependent $C P$ asymmetries in the $J / \psi K_{S / L}$ and $\phi K_{S / L}$ actually measure different quantities. Whereas the former measures $\sin (2 \beta)$, the latter measures $\sin (2 \beta+2 \chi)$. Since $\chi$ is very small in the $\mathrm{SM}$, these two measurements are expected to be identical, but in the models where $\chi$ can take larger values, these measurements have to be considered as independent ones.

Thus, in the VdQ model, the measurements of the $C P$ asymmetries in these two decays ${ }^{12}$ imply

$$
\begin{aligned}
& \sin \left(2 \beta_{\psi K}\right)=\sin (2 \tilde{\beta}) \\
& \sin \left(2 \beta_{\phi K}\right)=\sin (2 \tilde{\beta}+2 \tilde{\chi}) .
\end{aligned}
$$

Since within the SM the difference between the asymmetries in $B^{0} \rightarrow J / \psi K_{S}$ and $B^{0} \rightarrow \phi K_{S}$ is expected to be

$$
\left|\sin \left(2 \beta_{\psi K}\right)-\sin \left(2 \beta_{\phi K}\right)\right| \leq \sin (2 \chi) \approx \mathcal{O}\left(\lambda^{2}\right),
$$

if this value shows any significant deviation from the SM prediction, then it may reveal new physics effects. The difference between these two measurements in VdQ puts bounds on the value of $\tilde{\chi}$. Although it is consistent with zero at the moment, more data on $\phi K_{S / L}$ will reduce the errors, opening up a way for not only the detection of new physics, but also for the direct measurement of a new physics quantity. If this measurement coincides with the one through the $B_{s}-\bar{B}_{s}$ mixing, it will be a strong signal for the VdQ model.

We note that supposing the mode $B^{0} \rightarrow \phi K_{S}$ reveals new physics effects, one might expect that the other modes having the same internal quark level process $b \rightarrow s \bar{s} s\left(e . g ., B^{0} \rightarrow \eta^{\prime} K_{S}\right)$ would reveal similar effects ${ }^{15}$. Interestingly, the recent measurements of $\mathrm{CP}$ asymmetries in $B^{0} \rightarrow \eta^{\prime} K_{S}$ by Belle? agree well with the results of $\sin \left(2 \beta_{\psi_{K}}\right)$ given in $\mathrm{Eq}(48)$. However, as is well known, the strong interaction physics behind the decay $B^{0} \rightarrow \eta^{\prime} K_{S}$ is not well understood yet due to the uncertainties from anomalous gluonic contributions, intrinsic charm contents, involvements of spectator quark, etc., so we cannot yet draw any definite conclusions concerning any new physics effects in this decay.

\section{Electroweak Penguin and Leptophobic $Z^{\prime}$ Model}

Since in the standard model (SM) the flavor changing neutral current (FCNC) processes appear at the quantum level with suppression factors by small electroweak gauge coupling, CKM matrix elements, and loop momenta, they are very sensitive to probe new physics (NP) beyond the SM which have an enhancement factor in the coupling or have tree-level FCNCs.

The decay of $B$ mesons accumulated largely at asymmetric $B$-factories and Tevatron give an opportunity to probe NP models via the rare $B$ decays induced 
by FCNCs. Recently, among several sources for FCNCs in the $B$ decays, the electroweak (EW) penguin operators have drawn much interest. For example, the QCD penguin dominant $B \rightarrow K \pi$ decays appear to be very interesting since branching ratios (BRs) and mixing-induced $\mathrm{CP}$ asymmetry allow much room for large NP contribution, especially in the EW penguin sector ${ }^{16,17}$.

Most of models contributing to the EW penguin sector have a severe constraint from the $b \rightarrow s \gamma$ decay. While, models such as the $Z^{\prime}$ model are free from such constraints although they predict the EW penguin contributions. In order to probe such NP models, one must resort to nonleptonic decays or very rare process $B \rightarrow$ $M \nu \bar{\nu}\left(M=\pi, K, \rho, K^{*}\right)$. However, nonleptonic decays might be inefficient since they suffer from large hadronic uncertainties and EW penguins contributions are subdominant in nonleptonic decays.

Recently, D $\varnothing^{18}$ and CDF ${ }^{19}$ Collaborations at Fermilab Tevatron have reported the first observation of the mass difference $\Delta m_{s}$ in the $B_{s}^{0}-\bar{B}_{s}^{0}$ system which induced by the $b \rightarrow s$ FCNC:

$$
\begin{aligned}
& \text { DØ : } 17 \mathrm{ps}^{-1}<\Delta m_{s}<21 \mathrm{ps}^{-1} \text { (90\% C.L.), } \\
& \mathrm{CDF}: \Delta m_{s}=17.33_{-0.21}^{+0.42} \text { (stat.) } \pm 0.07 \text { (syst.) } \mathrm{ps}^{-1} \text {. }
\end{aligned}
$$

These measurements may give strong constraints on the NP models, which predict $b \rightarrow s$ FCNC transitions ${ }^{20,21}$.

In the present work, we focus on the leptophobic $Z^{\prime}$ model motivated from the flipped $\mathrm{SU}(5)$ or string-inspired $E_{6}$ models as a viable NP model. In Sec. 4.1, we briefly introduce the leptophobic $Z^{\prime}$ model. Section 4.2 deals with $B \rightarrow M \nu \bar{\nu}(M=$ $\left.\pi, K, \rho, K^{*}\right)$ decays within the leptophobic $Z^{\prime}$ model. We investigate implications of $\Delta m_{s}$ measurements on this model in Sec. 4.3.

\subsection{Leptophobic $Z^{\prime}$ model and $F C N C$}

In many new physics scenarios containing an additional $U(1)^{\prime}$ gauge group at the low energy, the new neutral gauge boson $Z^{\prime}$ would have a property of leptophobia, which means that the $Z^{\prime}$ boson does not couple to the ordinary SM charged leptons. In flipped $\mathrm{SU}(5) \times \mathrm{U}(1)$ scenario ${ }^{22}$, leptophobia of the $Z^{\prime}$ boson can be given naturally because the neutrino is subject to the different representation with the charged leptons. Another scenario for leptophobia can be found in the $E_{6}$ model with kinetic mixing, where in this model leptophobia is somewhat accidental. After breaking of the $E_{6}$ group, the low energy effective theory contains an extra $\mathrm{U}(1)^{\prime}$ which is a linear combination of $\mathrm{U}(1)_{\psi}$ and $\mathrm{U}(1)_{\chi}$ with a $E_{6}$ mixing angle $\theta^{23}$. Then, the general interaction Lagrangian of fermion fields and $Z^{\prime}$ gauge boson can be written as

$$
\mathcal{L}_{\mathrm{int}}=-\lambda \frac{g_{2}}{\cos \theta_{W}} \sqrt{\frac{5 \sin ^{2} \theta_{W}}{3}} \bar{\psi} \gamma^{\mu}\left(Q^{\prime}+\sqrt{\frac{3}{5}} \delta Y_{S M}\right) \psi Z_{\mu}^{\prime},
$$


where the ratio of gauge couplings $\lambda=g_{Q^{\prime}} / g_{Y}$, and $\delta=-\tan \chi / \lambda^{23}$. Since the general fermion- $Z^{\prime}$ couplings depend on two free parameters, $\tan \theta$ and $\delta$, effectively, the $Z^{\prime}$ boson can be leptophobic within an appropriate embedding of the SM particles ${ }^{23,24}$.

Assuming $V_{L}^{d}=1$ in the $E_{6}$ model and flipped SU(5) model, only $Z^{\prime}$-mediating FCNCs in the right-handed down-type quarks survive. Then, one can get the FCNC Lagrangian for the $b \rightarrow q(q=s, d)$ transition ${ }^{25}$

$$
\mathcal{L}_{\mathrm{FCNC}}^{Z^{\prime}}=-\frac{g_{2}}{2 \cos \theta_{W}} U_{q b}^{Z^{\prime}} \bar{q}_{R} \gamma^{\mu} b_{R} Z_{\mu}^{\prime},
$$

where all the theoretical uncertainties including the mixing parameters are absorbed into the coupling $U_{q b}^{Z^{\prime}}$. The coupling $U_{s b}^{Z^{\prime}}$ has in general CP violating complex phase, which we denote as $\phi_{s b}^{Z^{\prime}}$. We note that the leptophobic $Z^{\prime}$ boson is not well constrained by experiments including the charged leptons such as $b \rightarrow s \ell^{+} \ell^{-}$or $B_{(s)} \rightarrow \ell^{+} \ell^{-}$, while the typical new physics models are strongly constrained by such experiments.

\subsection{Exclusive $B \rightarrow M \nu \bar{\nu}$ Decays}

In this section, we consider the $B \rightarrow M \nu \bar{\nu}$ decays in the leptophobic $Z^{\prime}$ model. The $B \rightarrow M \nu \bar{\nu}$ decays are measured via the scalar or vector meson with the missing energy signal.

Theoretical estimates for BRs of the $B \rightarrow M \nu \bar{\nu}$ decays in the SM are $0.22_{-0.17}^{+0.27}$, $5.31_{-1.03}^{+1.11}, 0.49_{-0.38}^{+0.61}$, and $11.15_{-2.70}^{+3.05}$ in units of $10^{-6}$, respectively. While experiments by the Belle and BaBar Collaborations have reported only upper limits on BRs of $B \rightarrow K \nu \bar{\nu}$ and $B \rightarrow \pi \nu \bar{\nu}$ decays ${ }^{27,28}$, where the experimental bounds are about 7 times larger than the SM expectation for the $K$ production and much larger by an order of $10^{3}$ for the $\pi$ production.

The leptophobic $Z^{\prime}$ model can yield same signals as $B \rightarrow K \nu_{\mathrm{SM}} \bar{\nu}_{\mathrm{SM}}$ at detectors via the production of a pair of right-handed neutrinos instead of the ordinary SM neutrinos. In Fig. 3, we present our predictions for the BRs in the leptophobic $Z^{\prime}$ model as a function of the effective coupling $\left|U_{q b}^{Z^{\prime}}\right|$, where the mass of the $Z^{\prime}$ boson is assumed to be $700 \mathrm{GeV}$. The solid and dotted lines represent the estimates in the SM and the current experimental bounds, respectively. The dashed line denotes the expected BRs in the leptophobic $Z^{\prime}$ model. In spite that we choose a specific mass for the $Z^{\prime}$ boson, the present analysis can be easily translated through the corresponding changes in the effective coupling $\left|U_{q b}^{Z^{\prime}}\right|$ for different $Z^{\prime}$ boson mass. We extract the following constraints for the FCNC couplings from Fig. 3

$$
\left|U_{s b}^{Z^{\prime}}\right| \leq 0.29, \quad\left|U_{d b}^{Z^{\prime}}\right| \leq 0.61
$$

for $B \rightarrow K \nu \bar{\nu}$ and $B \rightarrow \pi \nu \bar{\nu}$ decays, respectively ${ }^{25}$. The present exclusive mode gives more stringent bounds on the leptophobic FCNC coupling compared with the inclusive $b \rightarrow s \nu \bar{\nu}$ decay ${ }^{24}$. 


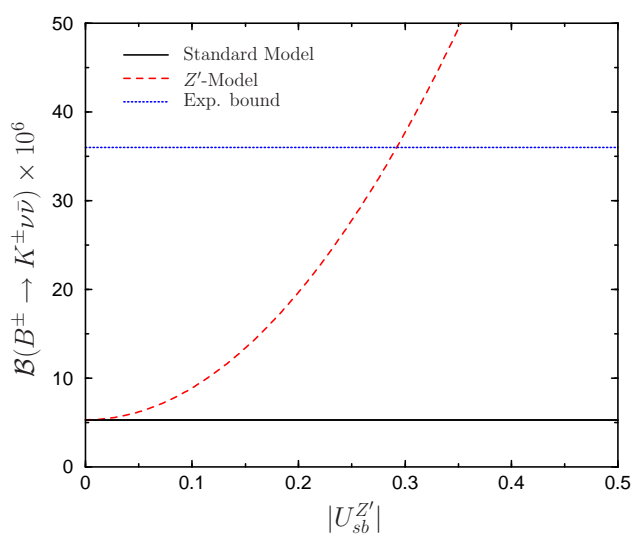

(a)

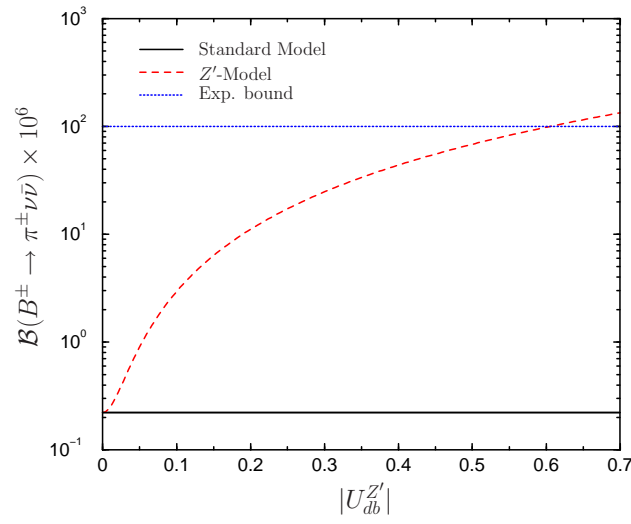

(b)

Fig. 3. Branching ratios for (a) $B^{ \pm} \rightarrow K^{ \pm} \nu \bar{\nu}$ and (b) $B^{ \pm} \rightarrow \pi^{ \pm} \nu \bar{\nu}$, where $\nu$ can be the ordinary SM neutrinos or right-handed neutrinos.

Recently, the Belle Collaboration has reported upper limits on the production of the $K^{*}$ meson with the missing energy signal at the $B$ decay where its BR is expected to be about 3 times larger than that of the scalar meson production in the $\mathrm{SM}^{26}$. It provides the constraint on the FCNC coupling

$$
\left|U_{s b}^{Z^{\prime}}\right| \leq 0.66
$$

which is larger than that in Eq. (53). At the super- $B$ factory, all four decay modes $B \rightarrow M \nu \bar{\nu}$ would be well measured and give more stringent bounds on the FCNC couplings.

The exclusive modes are much easier at the experimental detection than the inclusive ones. However, the exclusive modes have inevitable large theoretical uncertainties from hadronic transition form factors. In order to reduce hadronic uncertainties, one can take ratios for $\mathcal{B}(B \rightarrow M \nu \bar{\nu})$ to $\mathcal{B}(B \rightarrow M e \nu)$ for $M=\pi, \rho$ mesons ${ }^{25}$.

\section{3. $B_{s}^{0}-\bar{B}_{s}^{0}$ Mixing}

The $Z^{\prime}$-exchanging $\Delta B=\Delta S=2$ tree diagram contributes to the $B_{s}^{0}-\bar{B}_{s}^{0}$ mixing ${ }^{29}$. The mass difference $\Delta m_{s}$ of the mixing parameters then read

$$
\begin{gathered}
\Delta m_{s}=\Delta m_{s}^{\mathrm{SM}}\left|1+R e^{2 i \phi_{s b}^{Z^{\prime}}}\right| \\
R \equiv \frac{2 \sqrt{2} \pi^{2}}{G_{F} M_{W}^{2}\left(V_{t b} V_{t s}^{*}\right)^{2} S_{0}\left(x_{t}\right)} \frac{M_{Z}^{2}}{M_{Z^{\prime}}^{2}}\left|U_{s b}^{Z^{\prime}}\right|^{2}=1.62 \times 10^{3}\left(\frac{700 \mathrm{GeV}}{M_{Z^{\prime}}}\right)^{2}\left|U_{s b}^{Z^{\prime}}\right|^{2}
\end{gathered}
$$






(a)

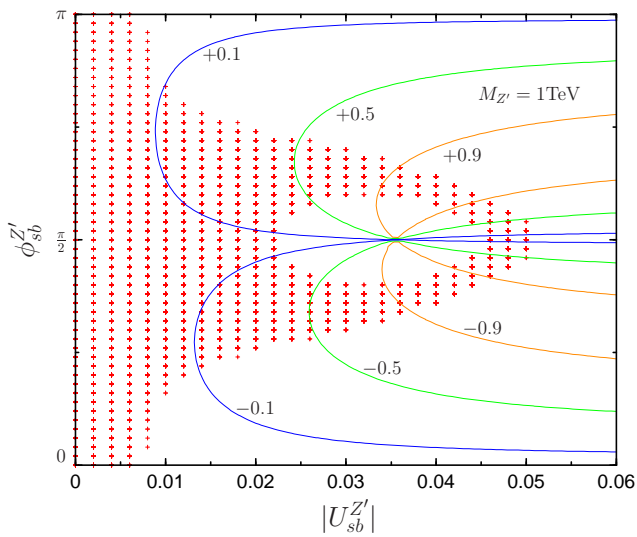

(b)

Fig. 4. The allowed region in $\left(\left|U_{s b}^{Z^{\prime}}\right|, \phi_{s b}^{Z^{\prime}}\right)$ plane for (a) $M_{Z^{\prime}}=700 \mathrm{GeV}$ and (b) $M_{Z^{\prime}}=1 \mathrm{TeV}$. We used $(\mathrm{HP}+\mathrm{JL}) \mathrm{QCD}$ result for the hadronic parameter. Constant contour lines for the time dependent CP asymmetry $S_{\psi \phi}$ in $B_{s} \rightarrow J / \psi \phi$ are also shown.

In Figs. 4, the allowed region in $\left(\left|U_{s b}^{Z^{\prime}}\right|, \phi_{s b}^{Z^{\prime}}\right)$ plane is shown. We obtain

$$
\left|U_{s b}^{Z^{\prime}}\right| \leq 0.0055 \quad \text { for } \mathrm{M}_{\mathrm{Z}^{\prime}}=700 \mathrm{GeV}
$$

for $\phi_{s b}^{Z^{\prime}}=0$. This bound is about two orders of magnitude stronger than (53) obtained from exclusive semileptonic $B \rightarrow M \nu \bar{\nu}$ decays.

The holes appear because they predict too small $\Delta m_{s}$. For a given $M_{Z^{\prime}}$ we can see that large $\mathrm{CP}$ violating phase can enhance the allowed coupling $\left|U_{s b}^{Z^{\prime}}\right|$ up to almost factor 10. This shows the importance of the role played by CP violating phase even in CP conserving observable such as $\Delta m_{s}$. As can be seen from Fig. $3(\mathrm{~b})$, irrespective of its phase $\phi_{s b}^{Z^{\prime}}$ value

$$
\left|U_{s b}^{Z^{\prime}}\right| \leq 0.051 \quad \text { for } \mathrm{M}_{\mathrm{Z}^{\prime}}=1 \mathrm{TeV} \text {. }
$$

The CP violating phase in $B_{s}^{0}-\bar{B}_{s}^{0}$ mixing amplitude can be measured at LHC in near future through the time-dependent $\mathrm{CP}$ asymmetry in $B_{s} \rightarrow J / \psi \phi$ decay

$$
\frac{\Gamma\left(\bar{B}_{s}^{0}(t) \rightarrow J / \psi \phi\right)-\Gamma\left(B_{s}^{0}(t) \rightarrow J / \psi \phi\right)}{\Gamma\left(\bar{B}_{s}^{0}(t) \rightarrow J / \psi \phi\right)+\Gamma\left(B_{s}^{0}(t) \rightarrow J / \psi \phi\right)} \equiv S_{\psi \phi} \sin \left(\Delta m_{s} t\right) .
$$

We note that although the final states are not CP-eigenstates, the time-dependent analysis of the $B_{s}^{0} \rightarrow J / \psi \phi$ angular distribution allows a clean extraction of $S_{\psi \phi}{ }^{31}$. In the SM, $S_{\psi \phi}$ is predicted to be very small, $S_{\psi \phi}^{\mathrm{SM}}=-\sin 2 \beta_{s}=0.038 \pm 0.003$ $\left(\beta_{s} \equiv \arg \left[\left(V_{t s}^{*} V_{t b}\right) /\left(V_{c s}^{*} V_{c b}\right)\right]\right)$. If NP has an additional CP violating phase $\phi_{s b}^{Z^{\prime}}$, however, the experimental value of

$$
S_{\psi \phi}=-\sin \left[2 \beta_{s}+\arg \left(1+R e^{2 i \phi_{s b}^{Z^{\prime}}}\right)\right]
$$


would be significantly different from the SM prediction. Constant contour lines for $S_{\psi \phi}$ are also shown in Figs. 4. We can see that even with the strong constraint from the present $\Delta m_{s}$ observation, large $S_{\psi \phi}$ are still allowed.

\section{Acknowledgements}

CSK is supported in part by CHEP-SRC and in part by the KRF Grant funded by the Korean Government (MOEHRD) No. KRF-2005-070-C00030.

\section{References}

1. N. Cabibbo, Phys. Rev. Lett. 10 (1963) 531; M. Kobayashi and T. Maskawa, Prog. Theor. Phys. 49 (1973) 652.

2. G. Barenboim, F.J. Botella and O Vives, Nucl. Phys. B 613 (2001) 285; T. Yanir, JHEP 0206 (2002) 044.

3. M. Bando and T. Kugo, Prog. Theor. Phys. 101 (1999) 1313; M. Bando, T. Kugo and K. Yoshioka, Prog. Theor. Phys. 104 (2000) 211.

4. N. Arkani-Hamed, S. Dimopoulos and G. Dvali, Phys. Lett. B429 (1998) 263; L. Randall and R. Sundrum, Phys. Rev. Lett. 83 (1999) 3370.

5. H. Davoudiasl, J. L. Hewett and T. G. Rizzo, Phys. Lett. B473 (2000) 43; T. Gherhetta and A. Pomarol, Nucl. Phys. B586 (2000) 141; F. del Aguila, M. Perez-Victoria and J. Santiago, Phys. Lett. B492 (2000) 98; J. L. Hetwett, F. J. Petriello and T. G. Rizzo, JHEP 0209 (2002) 030; C. S. Kim, J. D. Kim and J. H. Song, Phys. Rev. 67 (2003) 015001 [arXiv:hep-ph/0204002].

6. L. -L. Chau and W. -Y. Keung, Phys. Rev. Lett. 53, 1802 (1984).

7. L. Wolfenstein, Phys. Rev. Lett. 51, 1945 (1983).

8. J. D. Bjorken and I. Dunietz, Phys. Rev. D 36, 2109 (1987); C. Hamzaoui, Phys. Rev. Lett. 61, 35 (1988); G. C. Branco and L. Lavoura, Phys. Lett. B 208, 123 (1988); Phys. Rev. D 38, 2295 (1988); C. Jarlskog and R. Stora, Phys. Lett. B 208, 268 (1988); A. Campa, C. Hamzaoui and V. Rahal, Phys. Rev. D 39, 3435 (1989).

9. G. C. Branco, L. Lavoura, and J. P. Silva, CP violation, The International series of monographs on physics-103, Oxford Science Publications, Oxford, UK, 1999.

10. C. S. Kim, J. Rosner and C. P. Yuan, Phys. Rev. D 42, 96 (1990); G. Buchalla, A. J. Buras, M. E. Lautenbacher, Rev. Mod. Phys. 68, 1125 (1996); R. N. Cahn, M. P. Worah, Phys. Rev. D 60, 076006 (1999); A. Ali and D. London, Eur. Phys. J. C 9, 687 (1999); A. Buras, F. Parodi, and A. Stocchi, hep-ph/0207101; H. Abele, S. Baessler, D. Dubbers, J. Reich Nucl. Phys. A 663, 947 (2000); F. Parodi, Nucl. Phys. Proc. Suppl. bf 99B, 166 (2001).

11. A. Höcker, H. Lacker, S. Laplace (Orsay, LAL), F. Le Diberder, Eur. Phys. J. C 21, 225 (2001).

12. E. Barberio et al. [HFAG], arXiv:hep-ex/0603003.

13. W.-M. Yao et al. [Particle Data Group Collaboration], Journal of Physics G 33 (2006) 1.

14. F. del Aguila, M. Perez-Victoria and J. Santiago, JHEP 0009 (2000) 011 [arXiv:hep$\mathrm{ph} / 0007316]$.

15. B. Dutta, C. S. Kim and Sechul Oh, Phy. Rev. Lett. 90 (2003) 011801 [arXiv:hep$\mathrm{ph} / 0208226]$.

16. S. Baek, P. Hamel, D. London, A. Datta, and D. A. Suprun, Phys. Rev. D 71, 057502 (2005) [arXiv:hep-ph/0412086]; C. S. Kim, S. Oh, and C. Yu, Phys. Rev. D 72, 
074005 (2005) [arXiv:hep-ph/0505060]; S. Baek, JHEP 0607, 025 (2006) [arXiv:hep$\mathrm{ph} / 0605094]$.

17. Y. L. Wu, Y. F. Zhou, and C. Zhuang, arXiv:hep-ph/0609006 and references therein.

18. V. M. Abazov et al. [D0 Collaboration], Phys. Rev. Lett. 97, 021802 (2006) [arXiv:hepex/0603029].

19. A. Abulencia et al. [CDF - Run II Collaboration], Phys. Rev. Lett. 97, 062003 (2006) [arXiv:hep-ex/0606027].

20. S. Baek, JHEP 0609, 077 (2006) [arXiv:hep-ph/0605182]; R. Arnowitt, B. Dutta, B. Hu, and S. Oh, Phys. Lett. B 641, 305 (2006) [arXiv:hep-ph/0606130].

21. P. Langacker and M. Plumacher, Phys. Rev. D 62, 013006 (2000) [arXiv:hepph/0001204]; S. Chang, C. S. Kim and J. Song, JHEP 0702, 087 (2007) [arXiv:hep$\mathrm{ph} / 0607313]$.

22. J. L. Lopez and D. V. Nanopoulos, Phys. Rev. D 55, 397 (1997) [arXiv:hep$\mathrm{ph} / 9605359]$.

23. T. G. Rizzo, Phys. Rev. D 59, 015020 (1999) [arXiv:hep-ph/9806397].

24. K. Leroux and D. London, Phys. Lett. B 526, 97 (2002) [arXiv:hep-ph/0111246].

25. J. H. Jeon, C. S. Kim, J. Lee, and C. Yu, Phys. Lett. B 636, 270 (2006) [arXiv:hep$\mathrm{ph} / 0602156]$.

26. K. Abe et al. [Belle Collaboration], arXiv:hep-ex/0608047.

27. K. Abe et al. [Belle Collaboration], arXiv:hep-ex/0507034.

28. B. Aubert et al. [BABAR Collaboration], Phys. Rev. Lett. 94, 101801 (2005) [arXiv:hep-ex/0411061].

29. S. Baek, J. H. Jeon, and C. S. Kim, Phys. Lett. B 641, 183 (2006) [arXiv:hep$\mathrm{ph} / 0607113]$

30. S. Aoki et al. [JLQCD Collaboration], Phys. Rev. Lett. 91, 212001 (2003) [arXiv:hepph/0307039]; A. Gray et al. [HPQCD Collaboration], Phys. Rev. Lett. 95, 212001 (2005) [arXiv:hep-lat/0507015]; M. Okamoto, PoS LAT2005, 013 (2006) [arXiv:heplat/0510113]; P. Ball and R. Fleischer, arXiv:hep-ph/0604249.

31. A. S. Dighe, I. Dunietz, and R. Fleischer, Eur. Phys. J. C 6, 647 (1999) [arXiv:hepph/9804253]; I. Dunietz, R. Fleischer, and U. Nierste, Phys. Rev. D 63, 114015 (2001) [arXiv:hep-ph/0012219]. 